\title{
Intra-Abdominal Pressure and Abdominal Perfusion Pressure Early in Severe Acute Pancreatitis Misses the Forest for the Trees
}

\author{
Charles Joseph Miranda
}

Received: 10 June 2011 / Accepted: 14 September 2011 /Published online: 27 September 2011

(C) 2011 The Society for Surgery of the Alimentary Tract

I read with interest the results of Ke et al.'s ${ }^{1}$ recent study comparing intra-abdominal pressure (IAP) to abdominal perfusion pressure (APP) as a marker of severity in severe acute pancreatitis (SAP) published in this journal in April 2011. The rise in intra-abdominal pressure sometimes observed in severe acute pancreatitis continues to pose a clinical conundrum. Although the presence of abdominal compartment syndrome is usually treated with a decompressive laparotomy, ${ }^{2}$ advocating the same in acute pancreatitis would run contrary to the dogma that operations early in acute pancreatitis are associated with an increase in morbidity and mortality. ${ }^{3,4}$

The study purports to judge the efficacy of IAP and APP early in the onset of the disease as predictors of outcome. The authors agree that infection is a significant contributor to mortality after the early phase of the disease, ${ }^{5,6}$ but there is no evidence to indicate that this can be adequately detected $72 \mathrm{~h}$ after admission.

While I laud Ke et al.'s ${ }^{1}$ efforts to clarify the role of raised IAP in SAP, their conclusion that an intra-abdominal pressure $>15 \mathrm{mmHg}$ in SAP, within $72 \mathrm{~h}$ of admission, which is a valuable predictor of the outcome in SAP, should be approached with caution. In the absence of the consort data, we do not have information on patients who met the entry criteria but declined or were unable to participate in the study. Furthermore the definition of what constituted an infection was not stated (for example, Systemic Inflammatory Response Syndrome, positive blood cultures, fine needle aspiration and culture, or computed tomography evidence of free gas in the presence of pancreatic necrosis).
Even with the limitations described, demonstrating causality between the presence of raised IAP/reduced APP and the onset of infection requires the results from prospective controlled trial (i.e. incidence of infection in SAP patients with an IAP $>15$ is greater than the incidence of infection in SAP patients with an IAP $<15$ ). As it stands, it could be argued that a raised IAP in SAP is an epiphenomenon of the underlying disease process.

\section{References}

1. Ke L, Ni HB, Tong ZH, Li WQ, Li N, Li JS (2011) Intra-abdominal Pressure and Abdominal Perfusion Pressure: Which is a Better Marker of Severity in Patients with Severe Acute Pancreatitis. J Gastrointest Surg 15(8):1426-32

2. Chiara O, Cimbanassi S, Boati S, Bassi G (2011) Surgical management of abdominal compartment syndrome. Minerva Anestesiol 77 (4):457-62.

3. Tsiotos G, Luque-de Leon E, Sarr M. Long-term outcome of necrotizing pancreatitis treated by necrosectomy. $\mathrm{Br} \mathrm{J}$ Surg. 1998;85(12):1650-3.

4. Will U, Wegener C, Graf K, Wanzar I, Manger T, Meyer F. Differential treatment and early outcome in the interventional endoscopic management of pancreatic pseudocysts in 27 patients. World J Gastroenterol. 2006;12(26):4175-8.

5. Büchler MW, Gloor B, Müller CA, Friess H, Seiler CA, Uhl W. Acute necrotizing pancreatitis: treatment strategy according to the status of infection. Ann Surg. 2000;232(5):619-26.

6. Lytras D, Manes K, Triantopoulou C, Paraskeva C, Delis S, Avgerinos C, et al. Persistent early organ failure: defining the high-risk group of patients with severe acute pancreatitis? Pancreas. 2008;36 (3):249-54.
C. J. Miranda $(\bowtie)$

Hepatobiliary Surgery Unit, Manchester Royal Infirmary,

Manchester M13 9WL, UK

e-mail: cjmiranda2@gmail.com 